\title{
Spontaneous symmetry breaking and Lifshitz transition in bilayer graphene
}

\author{
Y. Lemonik, ${ }^{1}$ I.L. Aleiner, ${ }^{1,2}$ C. Toke ${ }^{3}$ and V.I. Fal'ko ${ }^{2,3}$ \\ ${ }^{1}$ Physics Department, Columbia University, New York, NY 1002\%, USA \\ ${ }^{2}$ Kavli Institute for Theoretical Physics China, CAS, Beijing 100190, China \\ ${ }^{3}$ Physics Department, Lancaster University, Lancaster, LA1 4YB, UK
}

\begin{abstract}
We derive the renormalization group equations describing all the short-range interactions in bilayer graphene allowed by symmetry and the long range Coulomb interaction. For certain range of parameters, we predict the first order phase transition to the uniaxially deformed gapless state accompanied by the change of the topology of the electron spectrum.
\end{abstract}

PACS numbers: 73.22.Pr, 73.21.-b

Introduction- The Lifshitz transition (LiTr) [1] is the simplest topological effect in physics of metals. It consists of the change of connectivity of isoenergetic surfaces, either as a function of electron density or external parameters, such as strain. As the change of the topology of the e.g. Fermi surface can not be continuous, all the observables in the system should experience singularities at the LiTr also known as a half-integer-order phase transition (PT). Alternatively, the reconstruction of the Fermi surface may occur via an underlaying spontaneous symmetry breaking PT. The observation of the LiTr in the bulk metals is an extremely challenging task: a variation of the Fermi level in metals requires doping which introduces disorder and obscures the transition, whereas application of strain requires high pressure experiments.

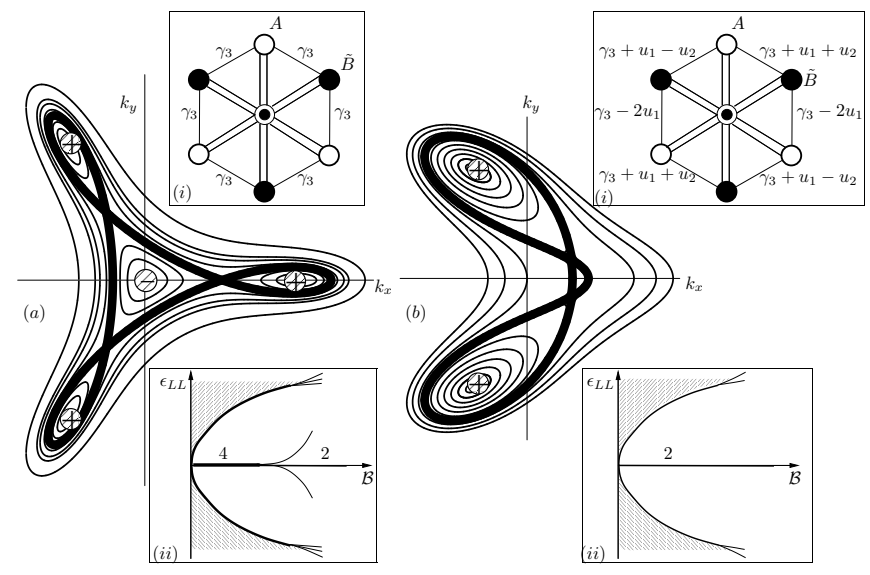

FIG. 1. Constant energy lines for the one particle spectrum in the BLG. LiTr as a function of density occurs when the Fermi level intersects the separatrix (bold line) for a) unbroken $\mathcal{C}_{6 v}$ symmetry of the graphene lattice; b) symmetry breaking $\mathcal{C}_{6 v} \rightarrow \mathcal{C}_{2 v}$. Circles mark Dirac points and \pm indicates Berry phases, $\phi_{B}= \pm \pi$. Insets shows the tight-binding cartoons for the band structure (i) and the schematic evolutions of the Landau levels in the magnetic field (ii) (the number indicates the degeneracy per one spin and valley).

Bilayer graphene (BLG) - a two-dimensional allotrope of carbon with a honeycomb lattice - is a potentially ideal system to study the LiTr [2]. A gapless low-energy elec- tronic structure of the conduction and valence bands near the Brillouin zone $(\mathrm{Bz})$ corners in Bernal stacked BLG has a parabolic dispersion $\epsilon_{ \pm} \approx \pm p^{2} / 2 m$ at intermediate energies determined by the intra- and interlayer hops between closest neighbors. Remarkably, the electronic wavefunctions accumulate the $\phi_{B}=2 \pi$ Berry phase as the momentum going along the loop encompassing $p=0$. This causes the double degeneracy (per one spin and one valley) of the zero-energy Landau level (LL) in the magnetic field. Those features, however, are not protected by the crystal symmetry. The parabolic dispersion is trigonally deformed at the lowest energies and $\epsilon=0$ state splits into four Dirac points: one in the $\mathrm{Bz}$ corner and three off-sets separated by momentum $2 m v_{3}$ due to the next-neighbor interlayer hopping, Fig. 1a (this separation is about $0.1 \%$ of the size of $\mathrm{Bz}$ ). The total $\phi_{B}$ is conserved so that the Dirac point in the $\mathrm{Bz}$ corner carries $\phi_{B}=-\pi$ and each of the off-sets $\phi_{B}=\pi$. This doubles the degeneracy of zero-energy LL at weak magnetic field.

Unlike in conventional metals, the LiTr in the BLG (from one to four Fermi lines) can be tuned by a small density variation controlled by a gate voltage. Suitable suspended BLG devices of sufficient quality for the LiTr studies have been fabricated $[3,4]$.

The central question of this Letter in the stability of the above Lifshitz transition against the effect of electronelectron interaction (EEI). There are two possibilities: (i) EEI does not break the symmetry leading to a quantitative renormalization of the band structure affecting, e.g. the density $n_{L i T r}=\left(2 / \pi^{2}\right)\left(m v_{3} / \hbar\right)^{2}$ corresponding to the LiTr; (ii) EEI does break the symmetry leading to a qualitative transformation of the spectrum - the number of the Dirac points is then determined by the reduced symmetry (contradicting scenarios were suggested in Refs. [5-7] ). Using the renormalization group (RG) treatment of the EEI problem we found that: (i) $n_{L i T r}$ is not renormalized; (ii) the most likely spontaneous symmetry breaking in BLG occurs by the generation of the asymmetric hopping in the effective Hamiltonian with the same symmetry as the effect of $A-\tilde{B}$ sublattice displacement [7], see Fig. 1b. For the gedanken experiment where $v_{3}$ is varied, the symmetry breaking occurs 
via first order quantum PT after which the spectrum remains gapless but two Dirac points are annihilated and two other persist and carry $\phi_{B}=\pi$; as the result, the degeneracy of the zero-energy Landau level is half that of the for unbroken $\mathcal{C}_{6 v}$ symmetry. The Berry phases control the degeneracy of the zero-energy LL which is visible via the Shubnikov-de Haas oscillations, and for some range of parameters the finite temperature $\mathrm{PT}$ is of the first order leading to bistabilities in transport.

The low energy model for the bilayer graphene is formulated in terms of the states close to $K$ and $K^{\prime}$ points of the Brillouin zone [2]. The Hamiltonian is

$$
\hat{\mathcal{H}}=\int d^{2} \mathbf{r} \Psi_{\sigma}^{\dagger}\left[\hat{h}_{0}+\hat{h}_{w}+\hat{h}_{c}+\hat{h}_{s r}\right] \Psi_{\sigma} .
$$

Hereinafter, the summation over repeated spin indices $\sigma= \pm 1 / 2$ is implied. Four component fermionic field $\Psi_{\sigma}=\left(\hat{\psi}_{\sigma}^{A, K}, \hat{\psi}_{\sigma}^{\tilde{B}, K} ; \hat{\psi}_{\sigma}^{\tilde{B}, K^{\prime}},-\hat{\psi}_{\sigma}^{A, K^{\prime}}\right)$ lives in the valley $\left(K K^{\prime}\right)$ and the sublattice $(A \tilde{B})$ spaces [2] (sublattices $A$ and $\tilde{B}$ belong to the different layers). All matrices acting in this four dimensional space are represented as direct product of the Pauli matrices $\hat{\tau}_{i}^{A \tilde{B}}, \hat{\tau}_{i}^{K K^{\prime}},(\mathrm{i}=0,1,2,3)$ :

$$
\hat{M}_{i}^{j} \equiv \hat{\tau}_{i}^{K K^{\prime}} \otimes \hat{\tau}_{j}^{A \tilde{B}}
$$

and $\hat{\tau}_{0} \cdots$ is the unit $2 \times 2$ matrix.

The kinetic energy is given by $\left(\hbar=1, k_{x, y}=-i \partial_{x, y}\right)$

$$
\hat{h}_{0}\left(k_{x, y}\right)=\left[\hat{M}_{3}^{1}\left(k_{x}^{2}-k_{y}^{2}\right)-2 \hat{M}_{3}^{2} k_{x} k_{y}\right] /(2 m) .
$$

Together with Eq. (1c), the trigonal warping term,

$$
\hat{h}_{w}\left(k_{x, y}\right)=-v_{3}\left[\hat{M}_{0}^{1} k_{x}+\hat{M}_{0}^{2} k_{y}\right],
$$

determines the spectrum in Fig. 1a.

The long-range Coulomb interaction,

$$
\hat{h}_{c}=\frac{e^{2}}{2} \int \frac{d^{2} \mathbf{r}^{\prime} \Psi_{\sigma^{\prime}}^{\dagger}\left(\mathbf{r}^{\prime}\right) \Psi_{\sigma^{\prime}}\left(\mathbf{r}^{\prime}\right)}{\left|\mathbf{r}-\mathbf{r}^{\prime}\right|},
$$

is the strongest in the system. However, due to the screening it does not scale and therefore does not describe any symmetry breaking by itself. The latter is captured by the scaling of the marginal short-range interaction

$$
\hat{h}_{s r}=(2 \pi / m) \sum_{i, j=0}^{3} g_{i}^{j} \hat{M}_{i}^{j}\left[\Psi^{\dagger} \hat{M}_{i}^{j} \Psi\right] .
$$

The couplings $g_{i}^{j}$ are not independent [8]. The $\mathcal{C}_{6 v}$ symmetry of the bilayer constrains

$$
\begin{aligned}
& g_{1}^{1}=g_{2}^{2}=g_{1}^{2}=g_{2}^{1}=g_{G} ; \quad g_{3}^{1}=g_{3}^{2}=g_{E_{1}} ; \\
& g_{1}^{3}=g_{2}^{3}=g_{E_{1}^{\prime \prime}} ; \quad g_{0}^{1}=g_{0}^{2}=g_{E_{2}} ; \quad g_{1}^{0}=g_{2}^{0}=g_{E_{2}^{\prime \prime}} ; \\
& g_{0}^{3}=g_{B_{1}} ; \quad g_{3}^{0}=g_{A_{2}} ; \quad g_{3}^{3}=g_{B_{2}},
\end{aligned}
$$

where subscripts indicate the irreducible representations of the extended point group, see e.g. Sec. III of Ref. [9]. For example, $E_{1}$ is two dimensional representation which does not change sign under $C_{2}$ rotation and describes the symmetry breaking shown on Fig. $1 \mathrm{~b}$, whereas $B_{2}$ is the one dimensional representation describing the breaking of the interlayer symmetry, $\mathcal{C}_{6 v} \rightarrow \mathcal{C}_{3 v}$.

The RG study of the model (1) is based upon the analysis of diagrams shown in Fig. 2. The Coulomb interaction apparently is the most relevant operator (i.e. its perturbative treatment leads to the linear rather than the logarithmic divergence). The screening of this interaction, see Fig. 2c, makes it marginal; its value is $\simeq 1 /[N \Pi(q, \omega)]$, ( $\Pi$ is the polarization operator). The formal justification for the approximation Fig. $2 \mathrm{c}$ is the $1 / N$ expansion - which we believe is applicable for $N=4$ - and the long wavelength limit. Note, that $g_{0}^{0}$ enters together with the Coulomb interaction potential so that it drops out, see Fig. 2c, and does not contribute to the running of the coupling constants. Other constants $g_{i}^{j}$ are assumed to be small and treated in a first loop approximation [10].

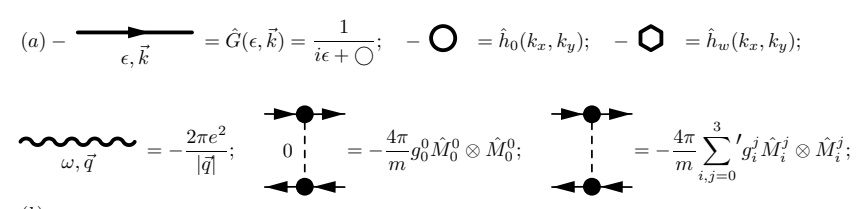

(b)

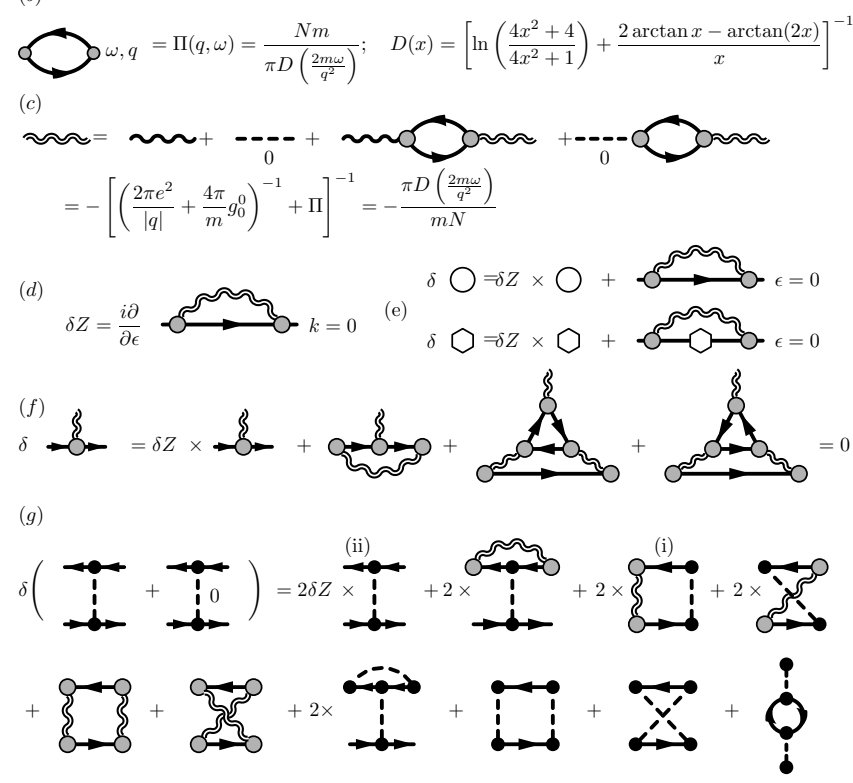

FIG. 2. Derivation of the RG equations. a) Definitions of the elements; b) Polarization operator; c) Screening of the Coulomb interaction; d,e) Renormalization of one particle spectrum; f) gauge invariance of the scalar vertex; g) renormalization of the short range interaction

Because the polarization operator does not have logarithmic divergences and all of the interactions are considered in the first loop, the details of the cut-off scheme are not important. On each step we will restrict the inter- 
nal momentum of the loop as $\mathcal{E}-d \mathcal{E}<k^{2} / 2 m(\mathcal{E}) \leq \mathcal{E}$. We, then, rescale $\psi \rightarrow(1+\delta Z / 2) \psi, \quad(\delta Z$ is defined on Fig. 2d) to keep the term $\partial_{\tau} \psi$ in the Matsubara equation intact. As a bonus, the scalar vertex is also not renormalized for the reason of gauge invariance, see Fig. 2f. Renormalizations of $m$ and $v_{3}$, see Fig. 2e, are given by[11]

$$
d \ln m / d \ell=-d \ln v_{3} / d \ell=-\alpha_{1} / N ; \alpha_{1} \approx-.078
$$

where $\ell \equiv \log \left(\mathcal{E}_{0} / \mathcal{E}\right)$, where $\mathcal{E}_{0} \simeq 0.3 \mathrm{eV}$ limits the applicability of the two-band model of bilayer graphene.

The possible symmetry breakings are described by the scaling of the short range interaction terms [11]:

$\frac{d g_{i}^{j}}{d \ell}=-\frac{\tilde{\alpha} \delta\left(E_{1}\right)_{i}^{j}}{N^{2}}-\frac{\alpha_{1} g_{i}^{j}}{N}-N B_{i}^{j}\left(g_{i}^{j}\right)^{2}-\sum_{k, l, m, n=0}^{3} C_{i ; k m}^{j ; l n} \tilde{g}_{k}^{l} \tilde{g}_{m}^{n}$

$\tilde{g}_{i}^{j} \equiv g_{i}^{j}\left(1-\delta_{i 0} \delta_{j 0}\right)+\delta_{i 0} \delta_{j 0} \alpha_{2} /(2 N), \alpha_{2} \approx .469$

where $\tilde{\alpha}=\alpha_{3}-\alpha_{2}^{2} / 16, \alpha_{3} \approx .066$, the symbol $\delta\left(E_{1}\right)_{i}^{j}$ is defined as $\delta\left(E_{1}\right)_{i=3}^{j=1,2}=1$ and $\delta\left(E_{1}\right)_{i}^{j}=0$ otherwise. The summation over repeated indices is not implied in Eq. (2b). The constants in Eq. (2b) are given by

$$
\begin{aligned}
B_{i}^{j} & =\frac{1}{16} \sum_{l=1,2} \operatorname{tr}\left\{\left[\hat{M}_{i}^{j}, \hat{M}_{3}^{l}\right]^{2}\right\} \\
C_{i ; k m}^{j ; l n} & =\frac{1}{32}\left\{\operatorname{tr}\left[\hat{M}_{i}^{j}\left[\hat{M}_{k}^{l}, \hat{M}_{m}^{n}\right]\right]\right\}^{2} \\
& +\frac{1}{64} \sum_{r=1,2}\left\{\operatorname{tr} \hat{M}_{3}^{r}\left(\hat{M}_{k}^{l} \hat{M}_{i}^{j} \hat{M}_{m}^{n}+\hat{M}_{m}^{n} \hat{M}_{i}^{j} \hat{M}_{k}^{l}\right)\right\}^{2} \\
& +\frac{\delta_{i k} \delta_{j l}}{4} \sum_{r=1,2} \operatorname{tr}\left\{\hat{M}_{m}^{n} \hat{M}_{3}^{r}\left[\hat{M}_{i}^{j}, \hat{M}_{3}^{r}\right] \hat{M}_{m}^{n} \hat{M}_{i}^{j}\right\} .
\end{aligned}
$$

Note that, Eqs. (2b) and (2c) respect symmetry (1g).

Equations (2) are the main technical result of this paper. They describe the evolution of all the band structure parameters and all short-range interactions terms allowed by symmetry in the leading logarithmic approximation. To compare with the existing literature: the RG treatment of Ref. [7] considers only two possible terms $\left(g_{E_{1}}\right.$, and $\left.g_{B_{1}}\right)$, treats the Coulomb interaction as shortrange and neglects the warping in the spectrum; meanfield treatment of Refs. [5, 6] corresponds to hardly justifiable taking into account only one [5] [(i) of Fig. 2g] or two [6] diagrams [(i,ii) of Fig. $2 \mathrm{~g}]$ with the subsequent projection on the $B_{2}$ representation.

$R G$ flow and non-broken symmetry - The density of electrons (or holes) at which the topology of the Fermi surface changes is found from Eqs. (1c) and (1d) as $n_{\text {LiTr }}=\left(2 / \pi^{2}\right)\left(m v_{3} / \hbar\right)^{2} \simeq 2 \times 10^{10} \mathrm{~cm}^{-2}$ (estimated with $m=0.035$ and $v_{3}=v_{3} \simeq 10^{7} \mathrm{~cm} / \mathrm{s}$ ). According to Eqs. (2a), the Coulomb part of the EEI does not renormalize $n_{L i T r}$ but affects the energy of the saddle points in the single-particle spectrum $\mathcal{E}_{L i T r} \equiv m v_{3}^{2} / 2$. The bare value of this energy can be estimated using the bilayer parameters $m, v_{3}$ quoted above as $\mathcal{E}_{L i T r} \simeq 1 \mathrm{meV}$.
The renormalized value is $\tilde{\mathcal{E}}_{L i T r}=\mathcal{E}_{L i T r}\left(\mathcal{E}_{0} / \mathcal{E}_{L i T r}\right)^{\frac{\alpha_{1}}{N}} \simeq$ $\mathcal{E}_{\text {LiTr }}\left(\mathcal{E}_{\text {LiTr }} / \mathcal{E}_{0}\right)^{0.02}$, such change is not observable.

$R G$ flow and symmetry breaking - The divergence of a coupling constant $g$. during the renormalization signals the symmetry breaking with the order parameter from the corresponding irreducible representation (a more complete classification, involving the the magnetic and gauge symmetries will be reported elsewhere [12]).

Let us assume with the short-range interactions on the energy scale $E_{0}$ are negligible, $g_{i}^{j}=0$. The constant term in Eq. (2b) means that this point is not fixed and couplings $g_{E_{1}}$ and $g_{B_{1}}$ (see Eq. $(1 \mathrm{~g})$ ) will flow away from this point. Ignoring $g_{B_{1}}$, we obtain an equation for $g_{E_{1}}$ :

$$
\begin{aligned}
& \frac{d g_{E_{1}}}{d \ell}=-\frac{c_{1}}{N(N+2)}-2(N+2)\left(g_{E_{1}}-c_{2}\right)^{2} \\
& c_{1} \approx\left(\frac{\alpha_{3}(N+2)}{N}-\frac{\left(\alpha_{2}-\alpha_{1}\right)^{2}}{8 N}\right) ; c_{2} \approx \frac{\alpha_{2}-\alpha_{1}}{4 N(N+2)} .
\end{aligned}
$$

Note that $c_{1}>0$, for $N>0$, and no fixed point exists; though $\alpha_{3}$ appears small, its neglecting would lead to a nontrivial fixed point $g_{E_{1}} \simeq 1 / N^{3}$. Solution of Eq. (3) is

$$
g_{E_{1}}(\ell)=c_{2}-\sqrt{\frac{c_{1}}{2 N(N+2)^{2}}} \cot \left[\sqrt{\frac{2 c_{1}}{N}}\left(\ell_{0}-\ell\right)\right]
$$

where $\ell_{0}$ is found from $g_{E_{1}}(\ell=0)=0$ : for $N=4$ $\ell_{0} \sim 7.1$. Inclusion of $g_{B_{1}}$ shifts the pole slightly so that $\left(g_{E_{1}} ; g_{B_{1}}\right) \propto\left(\ell_{0}-\ell\right)^{-1}(-1.67 ; 0.85)$.

This divergence implies a symmetry breaking at $\mathcal{E}_{E_{1}} \simeq$ $E_{0} e^{-7.1} \simeq 0.3 \mathrm{meV}[13]$. It is important to notice that $\mathcal{E}_{E_{1}}$ and $\mathcal{E}_{L i T r}$ turn out to be of the same order and, therefore, have to be considered together. A more accurate theoretical comparison of those two energy scales requires more detailed knowledge about the microscopic values of the initial interaction constants which is not available at this time. Therefore, we will discuss the possible PTs for an arbitrary value of $\Upsilon \equiv \mathcal{E}_{E_{1}} / \mathcal{E}_{L i T r}$.

Analysis of the phase transition- If $\Upsilon \lesssim 1$, the divergence of $g_{E_{1}}$ is terminated and the symmetry is not broken. The possible divergence of the coupling constant $g_{E_{1}}$ at $\Upsilon \gtrsim 1$ indicates the symmetry breaking and appearance of the anomalous averages comprising the irreducible representation $E_{1}$ of the group $\mathcal{C}_{6 v}$,

$$
u_{j}=(2 \pi / m)\left\langle\Psi_{\sigma}^{\dagger} \hat{M}_{3}^{j} \Psi_{\sigma}\right\rangle ; \quad j=1,2 .
$$

For studying the PT we have to consider the Landau free energy density. It must be of the form

$$
f=n_{L i T r} \mathcal{E}_{L i T r} \mathcal{F}_{\Upsilon}\left(\frac{u_{1}^{2}+u_{2}^{2}}{\mathcal{E}_{L i T r}^{2}} ; \frac{u_{1}^{3}-3 u_{1} u_{2}^{2}}{\mathcal{E}_{L i T r}^{3}}\right)
$$

for the symmetry and dimensionality reasons. At $\Upsilon<\Upsilon_{c}$ (here $\Upsilon_{c} \simeq 1$ ), function $\mathcal{F}(x, 0)$ has a local minimum at $x=0$ which, at $\Upsilon>\Upsilon_{c}$, turns to a maximum. The presence of the cubic invariant prescribed by $\mathcal{C}_{6 v}$ symmetry 


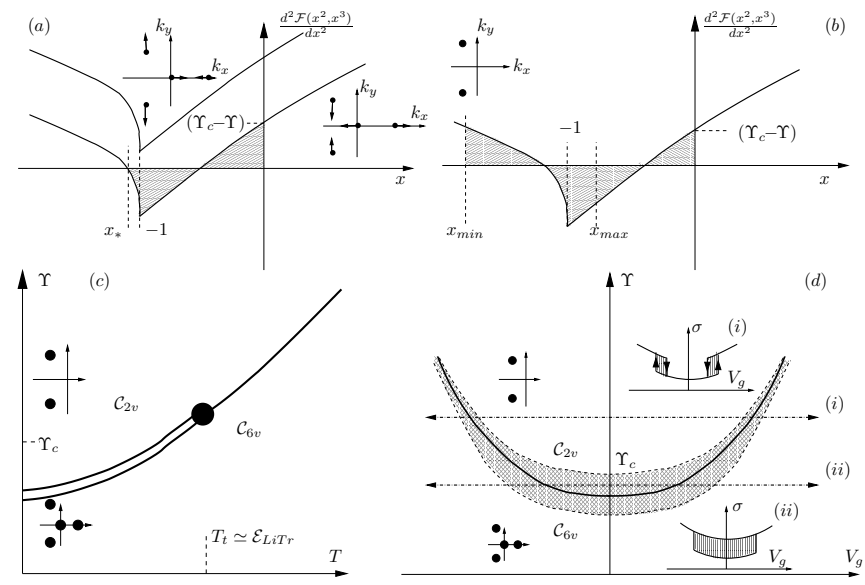

FIG. 3. a,b)The curvature of the mean-field energy along the steepest decent direction. The square root singularity is caused by the collision of the Dirac points shown on the inset. At $\Upsilon<\Upsilon_{1}<\Upsilon_{c}$ extra local minimum and maximum are formed (a), such as $\left|u_{1}^{\max }\right|>\mathcal{E}_{\text {LiTr }}$ (b), i.e. only two Dirac points remain. The total dashed area equals to zero. c,d) The schematic phase diagrams for the finite temperature (c) and for quantum (d) (controlled by the gate voltage $V_{g}$ ) PTs. The insets show predicted hysteretic (or slow noise) behavior (dashed areas) of the conductivity for the corresponding paths on the phase diagram.

signals that the zero temperature PT, under varying $\Upsilon$, can be only of the first order and occurs at $\Upsilon<\Upsilon_{c}$.

Now, we argue that the value of the order parameter in the ordered phase is such that the electron spectrum has two Dirac points, as in Fig. 1b. In the mean-field approximation, the one particle Hamiltonian reads

$$
\hat{H}=\hat{h}_{0}\left(k_{x, y}\right)+\hat{h}_{w}\left(k_{x, y}\right)-u_{1} \hat{M}_{3}^{1}-u_{2} \hat{M}_{3}^{2} .
$$

At $u_{1}=-\mathcal{E}_{\text {LiTr }}, u_{2}=0$ two Dirac points collide and disappear and the band structure of Fig. 1b is formed. At $u_{1}=3 \mathcal{E}_{L i T r}, u_{2}=0$ three Dirac points collide, and, once again, the spectrum with two Dirac points is formed.

The mean-field energy density is given by

$$
\begin{aligned}
& f_{M F}\left(u_{1}, u_{2}\right)=\left(\Upsilon_{c}-\Upsilon\right) \frac{m u_{i} u_{i}}{2 \pi} \\
& +\int \frac{d^{2} k}{\pi^{2}}\left[\epsilon(\mathbf{k})-\left.\epsilon(\mathbf{k})\right|_{u_{1,2}=0}-\left.\frac{u_{i} u_{j}}{2} \frac{\partial^{2} \epsilon(\mathbf{k})}{\partial u_{i} \partial u_{j}}\right|_{u_{1,2}=0}\right]
\end{aligned}
$$

where $\epsilon$ is the negative eigenvalue of $\hat{H}$, see Eq. (7), and the summation over the repeated indices $i, j=1,2$ is implied. The curvature of the energy density, found from Eq. (8), see Fig. 3, indicates that indeed $u_{1}$ formed during the PT transforms spectrum of Fig. 1a to that of Fig. 1b.

The corrections to the mean-field can not remove the singularity for the colliding Dirac points, as the Hamiltonian (7) at low energies is protected by symmetry. Therefore, we believe, that our conclusion about the number of Dirac points in ordered and disordered phases is more general than the mean-field derivation.
The conclusion about the first-order quantum phase transition have the important consequences for the finite temperature phase diagram, see Fig. 3c. At low temperatures $T \ll \mathcal{E}_{L i T r}$, the transition remains of the first order up to some tricritical temperature $T_{t}$, and at $T_{t}$ the transition is continuous and belongs to 3 states Potts model universality class [7]. The quantum phase transition can be studied as the function of density $n$, and the phase diagram is on Fig. 3d. The number of Dirac points in the ordered phase remains the same.

In conclusion, we investigated the interplay of the trigonal spectrum of the bare spectrum of the bilayer graphene with the electron-electron interaction. The derived $\mathrm{RG}$ equations allowed us to reveal the rich phase diagram [12] determined by the few (currently unknown) microscopic inputs. For a reasonably wide range of the initial conditions [13] we found $\mathcal{C}_{6 v} \rightarrow \mathcal{C}_{2 v}$ symmetry breaking and connected it with the change of the topology of the single particle spectrum. We predicted the quantum phase transition of the first order as a function of the electron density. Such a transition should be most readily observed in the hysteretic dependence of the conductivity on the gate controlled carrier density in the vicinity of the neutrality point.

This work was supported by US DOE contract No. DE- AC02-06CH11357 (I.A) and by EPSRC EP/G041954/1 (V.F.). We are grateful to A. Geim and A. Chubukov for valuable discussions.

[1] I. M. Lishitz, Zh. Exp. Teor. Fiz., 38, 1565 (1960) [Sov. Phys. JETP 11, 1130 (1960)]; A. A. Abrikosov, Fundamentals of the Theory of Metals. Elsevier, 1988.

[2] E. McCann \& V. Fal'ko, Phys. Rev. Lett. 96, 086805 (2006).

[3] B.E. Feldman, J. Martin, \& A. Yacoby, Nature Physics 5, 889 (2009).

[4] A. Geim (private communication).

[5] R. Nandkishore \& L. Levitov, arXiv:0907.5395v1.

[6] R. Nandkishore \& L. Levitov, arXiv:0907.5395v2, Phys. Rev. Lett., 104, 156803, (2010).

[7] O.Vafek \& K.Yang, Phys. Rev. B 81, 041401(R) (2010).

[8] I.L. Aleiner, D.E.Kharzeev, \& A.M.Tsvelik, Phys. Rev. B 76, 195415 (2007).

[9] D.M. Basko, Phys. Rev. B 78, 125418 (2008).

[10] For similar treatment in monolayer see Ref. [8] and J.E. Drut \& D.T. Son, Phys. Rev. B 77, 075115 (2008).

[11] Analytic expressions for coefficients $\alpha_{1,2,3}$ are $\left(\alpha_{1} ; \alpha_{2} ; \alpha_{3}\right)=\frac{1}{2 \pi} \int_{-\infty}^{\infty} \frac{d x D(x)}{\left(1+x^{2}\right)^{2}}\left(\frac{1-3 x^{2}}{\left(1+x^{2}\right)} ; 2 ; \frac{D(x)}{4}\right)$, where the function $D(x)$ is defined on Fig. $2 \mathrm{~b}$.

[12] Y. Lemonik, I.L. Aleiner, \& V.I. Fal'ko (in preparation).

[13] Line Eq. (4) is an unstable solution of Eq. (2b) and may be used only if the bare values of all other $g$. $\lesssim 1 / N$, so that they are still small at energy $\mathcal{E}_{1}$. All possible stable directions, their basins of attraction, and extremely rich phase diagram will be reported in Ref. [12]. 